\title{
Ultrasound transducer disinfection in emergency medicine practice
}

\author{
Riley Hoyer', Srikar Adhikari ${ }^{2}$ and Richard Amini ${ }^{2}$
}

\begin{abstract}
Background: External ultrasound transducer disinfection is common practice in medicine. Unfortunately, clinically significant organisms, such as methicillin-resistant Staphylococcus aureus (MRSA), Pseudomonas aeruginosa, and Klebsiella pneumonia spread throughout healthcare facilities via direct contact despite disinfection protocols. Ultrasound transducers and coupling gel provide potential vectors for pathogen transmission, especially in immunocompromised and high-risk patient populations. Our objective was to conduct a survey to investigate the variety of cleaning solutions or sanitary wipes used and evaluate current standard practice for transducer disinfection across emergency medicine training programs in the United States.

Findings: Eighty-three academic emergency medicine programs participated in this study. Eighty-seven percent $(95 \% \mathrm{Cl}$ 80-94\%) of responding programs do not have a mandated protocol or standard contact time for transducer disinfection. Ninety percent (95\% Cl 84-96\%) of institutions use disinfectant solution or disinfectant wipes, as the standard of practice, to cleanse ultrasound transducers after every use.

Conclusions: Currently, there is a great deal of variability with regard to non-endocavitary transducer disinfection protocols that seems to stem from the vast number of disinfectant products and ultrasound manufacturer disparate recommendations. In order to mitigate risk to patients and reduce health care costs linked to nosocomial infections; healthcare providers, ultrasound companies, and disinfectant manufacturers must develop a universal use disinfectant and a standard protocol for ultrasound device disinfection for noncritical device disinfection in the emergency department.
\end{abstract}

\section{Introduction}

External ultrasound (US) transducer disinfection is common practice in modern medicine. With the rising utility of US as a diagnostic procedure in the emergency room setting, patient contact with ultrasound transducers has clearly increased over recent years. Currently, no universal standard for external-use US transducer disinfection exists. Clinically significant organisms, such as methicillinresistant S. aureus (MRSA), P. aeruginosa, K. pneumonia spread throughout healthcare facilities via direct contact despite disinfection protocols [1]. US transducers and coupling gel provide potential vectors for pathogen transmission, especially in immunocompromised and high-risk patient populations [2]. In order to reduce nosocomial infection and improve disinfection practices, health-care

\footnotetext{
* Correspondence: richardamini@gmail.com

${ }^{2}$ Department of Emergency Medicine, The University of Arizona, PO Box 245057, Tucson, AZ 85724-5057, USA

Full list of author information is available at the end of the article
}

providers must direct their attention to the lack of uniformity in US transducer disinfection.

The Center for Disease Control and Prevention (CDC) classifies all non-endocavitary US transducers as noncritical devices by definition meaning this medical equipment does not directly contact mucosal surfaces or non-intact skin [3]. Non-endocavitary US transducers do not require high-level disinfection and/or protection of the transducer with sterile transducer-cover between uses to prevent bacterial contamination. However, these devices require low-level disinfection. According to the CDC, low-level disinfection consists of greater than 1-minute exposure time of device to the following solutions or wipes: ethyl alcohol (70-90\%), sodium hypochlorite (5.25-6.15\%), phenolic germicidal detergent, iodophor germicidal detergent, or quaternary ammonium germicidal solution [4]. A variety of germicidal techniques have been studied as lowlevel disinfection including the following: $>80{ }^{\circ} \mathrm{C} \mathrm{H}_{2} \mathrm{O}$, $0.5 \%$ accelerated hydrogen peroxide, and quaternary 
ammonia germicidal wipes $[5,6]$. In comparison, the CDC definition of high-level disinfection consists of 12-30 min of exposure time at $>20{ }^{\circ} \mathrm{C}$ with the following solutions: gultaraldehyde-based formulations ( $>2 \%)$, Ortho-phthalaldehyde (0.55\%), and hydrogen peroxide $(7.5 \%)$ [4].

After each use of an external ultrasound transducer, most institutions focus on two components of transducer disinfection: gross decontamination by removal of ultrasound gel, and low-level disinfection with germicidal wipes. Unfortunately, chemical components and recommended contact time vary between products. Commonly used germicidal wipes for US transducer disinfection include Super Sani-Cloth (isopropyl alcohol $55 \%$, aklyl ammonium compound $0.25 \%$ ), Sani-Cloth Plus (isopropanolol $14.85 \%$, quaternary ammonium $0.125 \%$ ), and Sani-Cloth HB (benzyl ammonium chlorides $0.07 \%$, quaternary ammonium $0.07 \%$ ). The Sani-Cloth manufacturer recommends a 2 min contact time for Super Sani-Cloth, 3 min contact time for Sani-Cloth Plus, and a $10 \mathrm{~min}$ contact time for Sani-Cloth HB [7-9]. Our objective was to conduct a survey to investigate the variety of cleaning solutions or sanitary wipes used and evaluate current standard practice for transducer disinfection across emergency medicine training programs in the United States.

\section{Methods}

This was a cross-sectional survey study conducted electronically using a questionnaire developed by the investigators. The study was approved per the university's process for approval of quality improvement projects involving humans. Respondents were from the Society for Academic Emergency Medicine (SAEM) ultrasound interest listserv. This listserv is comprised of ultrasound directors at academic emergency medicine programs primarily across the United States. A 5-item questionnaire (Additional file 1) on use of ultrasound transducer disinfection in the emergency department was created based on review of existing literature, knowledge of current practices, and discussions with experts in the field. Two emergency medicine (EM) physicians with expertise in point of care ultrasound reviewed the questionnaire for content validity. They evaluated all questions for relevance and clarity, as well as overall comprehensiveness of the questionnaire. The survey consisted of multiplechoice and free-text response questions regarding transducer disinfection including: current standard of practice in transducer disinfection, different disinfectant products, and duration of contact time between the transducer and disinfectant. The questionnaire was distributed via e-mail through the SAEM listserv. The email message included a link to the survey (Surveymonkey.com, Palo Alto, California, USA), as well as an optout option. The survey was collected anonymously.
Descriptive statistics was used to summarize the data using statistical analysis software (SAS) version 9.3 (Copyright, SAS Institute Inc., Cary, NC, USA). The survey responses are reported as the percentages of total respondents along with $95 \%$ confidence intervals.

\section{Results}

Eighty-three programs participated in this study. Eightyseven percent (95\% CI 80-94\%) of responding programs do not have a standard of practice for transducer contact time with disinfectant. At $90 \%$ (95\% CI 84-96\%) of the institutions, it is standard practice to use disinfectant solution or disinfectant wipes to cleanse ultrasound transducers after every use. Furthermore, 76 \% (95 \% CI 67-85 \%) of the institutions performed a brief wipe (less than $15 \mathrm{~s}$ ) as standard of practice for the recommended duration of contact time between the transducer and disinfectant. Only a small number of the surveyed institutions reported extended contact times for transducer disinfection: $9.6 \%$ (95\% CI 3-16\%) 1 min, 2.4 \% (95\% CI 1-6 \%) 2 min, $2.4 \%$ (95\% CI 1-6 \%) 3 min. For the purpose of ultrasound transducer disinfection, EM residency programs use a variety of products, which are summarized in Table 1. The most commonly used product was PDI Super SaniCloth wipes $33 \%$ (95 \% CI 23-43\%).

\section{Discussion}

Hospital-acquired infections in the United States present a significant risk for patient safety and place immense financial impact on the healthcare system. According to the Center for Disease Dynamics, Economics, and Policy (CDDEP), the United States spends 9.8 billion dollars on hospital-acquired infections annually [10]. Ultrasound transducers and coupling gel have the potential to spread hospital-acquired infections. Previous studies identified clinically significant organisms on ultrasound equipment and have implied that nocosomial infections can be spread by ultrasound equipment. One study demonstrated that $18 \%$ of ultrasound transducers were contaminated with clinically significant organisms including:

Table 1 Most commonly used disinfectant wipes and solution at different emergency departments

\begin{tabular}{lll}
\hline Disinfectant & \# of programs & Percentage $^{\text {a }}(95 \%$ Cl) \\
\hline PDI Super Sani-Cloth Wipe & 27 & $33 \%(23-43 \% \mathrm{Cl})$ \\
PDI Sani-Cloth Plus Wipe & 19 & $23 \%(14-32 \% \mathrm{Cl})$ \\
T-Spray Solution & 15 & $18 \%(10-26 \% \mathrm{Cl})$ \\
PDI Sani-Cloth HB Wipe & 14 & $17 \%(9-25 \% \mathrm{Cl})$ \\
Other $^{\mathrm{b}}$ & 15 & $18 \%(10-26 \% \mathrm{Cl})$ \\
\hline
\end{tabular}

${ }^{\mathrm{a}}$ Total number of participating programs $N=83$. ${ }^{\mathrm{b}}$ Including: Sani-Cloth Bleach wipe, Sani-Cloth Hands wipe, Oxivir Tb wipe, Transeptic solution, Sani-Cloth AF3 wipe, Isopropyl alcohol wipe, and CaviWipes 
Enterococci, S. aureus, Proteus mirabilis, Escherichia coli, Group B Streptococci, and Proteus vulgaris [11]. Another study reported $K$. penumoniae, resistant to third generation cephalosporins, contaminated ultrasound coupling gel and infected both adult female and neonatal patients in an obstetrics and gynecology department [12]. In 2013, Chittick et al. discovered P. aeurginosa, in ultrasound gel, as the source of infection in patients who received a transthoracic echocardiogram during hospitalization [13].

The probability of potential infection caused by ultrasound transducers varies in the literature. One study assessed transducer disinfection across all departments at a military academic institution and found that there was an insufficient number of transducers grossly contaminated to yield valid results [14]. In contrast, Sanz et al. reported that gross debris was removed from ultrasound transducers after use in only $58 \%$ of medical cases and only $33 \%$ of trauma cases [15]. Gross contaminants must be wiped away prior to utilizing a cleaning solution or germicidal wipe because contents of the ultrasound gel may inhibit the germicidal effects of the disinfectant. After removing gross contamination, a germicidal agent should be used to disinfect the transducer, transducer cable, and US machine. In our study, $90 \%$ of programs have standard practice for the use of germicidal wipes or solution for transducer disinfection after every use. Unfortunately, many of the programs adhere poorly to manufacturer recommended disinfectant contact times, with $76 \%$ of programs performing only a brief wipe (less than $15 \mathrm{~s}$ ) for transducer disinfection. Our data demonstrates that the most utilized germicidal wipe across academic institutions with EM residency programs in the United States is the PDI Super SaniCloth (33\% of programs). According to the manufacturer material safety data sheet (MSDS), the Super SaniCloth provides the greatest utility in the acute care setting as it requires a contact time of only $2 \mathrm{~min}$. In addition, it provides a broader spectrum of antimicrobial coverage than Sani-Cloth Plus germicidal wipes [8]. Our survey revealed that, only $15 \%$ of programs use at least a 1 min contact time as standard of practice for disinfection.

Unfortunately, manufacturers of commonly used ultrasound machines, the Philips Sparq and Zonare Z.one pro, do not recommend disinfection with PDI Super SaniCloth wipes $[16,17]$. The active chemicals for disinfection in PDI Super Sani-Cloth wipes can be damaging to ultrasound transducer surfaces and device surfaces including transducer footprint, screen, cables, and connectors. Comparing the utility of PDI Sani-Cloth Plus, T-Spray and PDI Sani-Cloth HB, both T-spray and PDI Sani-Cloth HB are recommended for transducer, cable and connector disinfection; while, PDI Sani-Cloth PLUS should only be used to disinfect the transducers. Our review of germicidal wipes and solutions demonstrates that, of the disinfectants reported in our survey, SaniCloth HB provides the greatest utility of low-level disinfectants because these products are recommended by Phillips to disinfect all ultrasound surfaces including monitors and screens and this disinfectant is effective against 100+ microrganisms. [16] However, the $10 \mathrm{~min}$ recommended contact time of Sani-Cloth $\mathrm{HB}$ creates a challenge for the use of this wipe in a busy emergency department [9]. For disinfectants that require extended contact times, such as Sani-Cloth HB, providers should be aware that reduced contact times, shorter than recommended by the manufacturer, can result in ineffective disinfection and spread of clinically significant microorganisms in the hospital setting. Further investigation should be focused on collaborations between ultrasound companies and disinfectant wipe manufacturers in order to establish a universal disinfectant wipe that can be used on all ultrasound machine components that is fast acting and has broad spectrum germicidal properties.

\section{Limitations}

This survey-based study has several limitations. Responder bias may have resulted in overrepresentation of programs with greater interest in ultrasound and perhaps greater expertise in transducer disinfection. Although the survey was pilot-tested, it was not validated; and a majority of the questions were closed-ended which might have introduced a response bias. As with any survey study, survey responses are limited by interpretation of questions and are vulnerable to error. Our survey was collected anonymously, as a result, we are unable to determine the exact demographics of our responders. We are unable to acertain the percentage of respondents from tertiary care centers, small community centers, or the specific application of this data to different levels of health care centers. Additionally, our survey was only distributed to academic institutions which limits the generalizability of our findings. Finally, as with any survey, survey responses are limited by interpretation of questions and are vulnerable to error.

\section{Conclusion}

Currently, there is a great deal of variability with regard to non-endocavitary, external transducer disinfection protocols at academic institutions in the United States. This variability seems to stem from the vast number of disinfectant products and ultrasound manufacturer disparate recommendations. In order to mitigate risk to patients and reduce healthcare costs linked to nosocomial infections, healthcare providers, ultrasound companies, and disinfectant manufacturers must develop a universal use disinfectant and a standard protocol for ultrasound device disinfection in the emergency department. 


\section{Additional file}

Additional file 1: Appendix A - Online Survey. (DOC 26 kb)

\section{Competing interest}

The authors declare that they have no competing interests.

\section{Authors' contributions}

RA created conceptualized the project and created the survey. SA participated in editing the survey and the manuscript. $\mathrm{RH}$ drafted the manuscripts, participated in coordinating the project, and created the reference list. All authors read and approved the final manuscript.

\section{Author details}

${ }^{1}$ College of Medicine, The University of Arizona, Tucson, AZ, USA

2Department of Emergency Medicine, The University of Arizona, PO Box 245057, Tucson, AZ 85724-5057, USA.

\section{Received: 7 January 2016 Accepted: 22 March 2016}

\section{Published online: 04 April 2016}

\section{References}

1. Miyague AH, Mauad FM, Martins WP, Benedetti AC, Ferreira AE, Mauad-Filho F. Ultrasound scan as a potential source of nosocomial and crossinfection: a literature review. Radiol Bras. 2015;48(5):319-23.

2. Schabrun S, Chipchase L, Rickard H. Are therapeutic ultrasound units a potential vector for nosocomial infection? Physiother Res Int. 2006;11(2):61-71.

3. Frazee BW, Fahimi J, Lambert L, Nagdev A. Emergency department ultrasonographic probe contamination and experimental model of probe disinfection. Ann Emerg Med. 2011;58(1):56-63.

4. Rutala W, Weber D. Guideline for Disinfection and Sterilization in Healthcare Facilities. [Internet]. Atlanta: Center for Disease Control and Prevention; 2008. Available from: http://www.cdc.gov/hicpac/pdf/guidelines/ Disinfection_Nov_2008.pdf

5. Savasci U, Oren NC, Akpak YK, et al. Comparison of probe disinfection procedures in routine ultrasonography: hot water versus antiseptic wiping Intern Med. 2014;53(19):2201-4.

6. Dancer SJ. Importance of the environment in meticillin-resistant Staphylococcus aureus acquisition: the case for hospital cleaning. Lancet Infect Dis. 2008;8(2):101-13.

7. Safety Data Sheet. Super Sani-Cloth [Internet]. Orangeburg: Nice-Park/PDI, Inc; 2015. Available from: https://www.conncoll.edu/media/website-media/ offices/ehs/envhealthdocs/Super_Sani-Cloth.pdf.

8. Safety Data Sheet. Sani-Cloth Plus [Internet]. Orangeburg: Nice-Park/PDI, Inc; 2015. Available from: http://pdihc.com/uploads/datasheets/ bdb86c7c1b27334c2a29b5ec14fe07b9.pdf.

9. Safety Data Sheet. Sani-Cloth HB. [Internet]. Orangeburg: Nice-Park/PDI, Inc; 2015. Available from: http://www.dentalcity.com/MSDS/76-300HB.pdf.

10. Zimlichman E, Henderson D, Tamir O, et al. Health care-associated infections: a meta-analysis of costs and financial impact on the US health care system. JAMA Intern Med. 2013;173(22):2039-46.

11. Patterson SL, Monga M, Silva JB, Bishop KD, Blanco JD. Microbiologic assessment of the transabdominal ultrasound transducer head. South Med J. 1996;89(5):503-4.

12. Gaillot $\mathrm{O}$, Maruéjouls $\mathrm{C}$, Abachin $\mathrm{E}$, et al. Nosocomial outbreak of Klebsiella pneumoniae producing SHV-5 extended-spectrum beta-lactamase, originating from a contaminated ultrasonography coupling gel. J Clin Microbiol. 1998;36(5):1357-60.

13. Chittick P, Russo V, Sims M, et al. An outbreak of Pseudomonas aeruginosa respiratory tract infections associated with intrinsically contaminated ultrasound transmission gel. Infect Control Hosp Epidemiol. 2013;34(8):850-3.

14. Lawrence MW, Blanks J, Ayala R, et al. Hospital-wide survey of bacterial contamination of point-of-care ultrasound probes and coupling gel. J Ultrasound Med. 2014:33(3):457-62.

15. Sanz GE, Theoret J, Liao MM, Erickson C, Kendall JL. Bacterial contamination and cleanliness of emergency department ultrasound probes. CJEM. 2011; 13(6):384-9.

16. Sparq Ultrasound System. Disinfectants and Cleaning Solutions. [Internet]. Andover: Philips Healthcare; 2014. Available from: http://www.healthcare.
philips.com/main/shared/Assets/Documents/Ultrasound/Products/ Transducers/Sparq 453561739702a.pdf/.

17. Zonare Transducers. Cleaning \& Disinfection. [Internet]. Mountain View: ZONARE Medical Systems Inc; 2013. Available from: http://www. pocustoronto.com/equipment/endocav/story_content/external_files/ Zonare\%20-\%20transducer\%20cleaning.pdf/.

\section{Submit your next manuscript to BioMed Central and we will help you at every step:}

- We accept pre-submission inquiries

- Our selector tool helps you to find the most relevant journal

- We provide round the clock customer support

- Convenient online submission

- Thorough peer review

- Inclusion in PubMed and all major indexing services

- Maximum visibility for your research

Submit your manuscript at www.biomedcentral.com/submit 